\title{
A Multiaccess Enabled Load Balancing in Cognitive Networks
}

\author{
Teemu Rautio and Jukka Mäkelä \\ VTT Technical Research Centre of Finland, \\ Kaitoväylä 1, FI-90571 Oulu, Finland \\ \{teemu.rautio, jukka.makela\} @vtt.fi
}

\begin{abstract}
During the last years, the increasing complexity of the communication networks and the continuously growing amount of data traffic has created great challenges for the network and service management. The Univerself project is currently designing a novel Unified Management Framework (UMF) consolidating autonomic and cognitive methods of the future Internet for business-driven service and network management. In this paper we describe our ongoing work with one of the validation scenarios for autonomous load balancing in a wireless multiaccess environment. In the proof-of-concept prototype, we concentrate on monitoring of network nodes and autonomous resource optimization by using a Distributed Decision Engine and a multiaccess capable access selection. In addition, we introduce the required key components and place them into the scenario. At the end of the paper, we draw up the future plans.
\end{abstract}

Keywords: autonomous network management, load balancing, distributed decision making, multi-access.

\section{Introduction}

The ever-increasing complexity of data networks has created great challenges for network and service management. Only in the wireless domain, the number of technologies, including i.e. GPRS, UMTS, HSPA, LTE, WiMAX and different WLAN technologies, together with a big number of employed services, creates an environment, where a huge amount of information available at different locations is rather a rule than an exception. The usage of this information is beneficial, and sometimes mandatory, for achieving the optimized usage of the network resources. Managing efficiently such a number of items all over networks and services is difficult, if not even impossible without any cognitive management solutions. On the other hand, by limiting observation scope too much, the end result might be of doing optimizations, which actually impair i.e. the overall network/service performance. Examples of concrete problems in information management include the functionalities, such as the collection of the data from varying type of sources and the efficient further processing of the data. An important functionality is the decision making based on the collected and processed information, for example, considering the optimization of the traffic load via certain wireless access points, as is in our case. One of the main goals of the optimizations is to provide a better end-user quality. 
In addition to the complexity problem, the amount of data traffic is continuously growing, especially in the wireless and mobile networks. According to Cisco [1], only in 2010, data traffic in mobile networks has grown 2.6-fold and the excepted to increase between 2010 and 2015 is as high as 26-fold. If such an explosive growth of traffic will be bound to distribute unequally among the network, it will lead to the situation where the network resources are used unequally and inefficiently. With the autonomous load balancing and network management solutions, the challenges for the data/traffic distribution in the future networks can be influenced significantly.

Univerself project [2] is designing a Unified Management Framework (UMF) [3] for widespread deployments of autonomics in communication networks and services. UMF is taking an approach, where the distributed decision making process and autonomous management can be handled under operator's supervision, and where different network domains, technologies as well as devices can be operated consistently.

This paper describes our on-going work on multiaccess capable load balancing in wireless domain as a part of the Univerself project. We have defined one validation scenario of UMF in order to bring autonomics to reality, and this scenario will be introduced hereafter. By following the guidelines of UMF, our approach for distributed resource management is defined. In addition, we depict the main components of our approach and our prototype, and place them into the scenario. Finally we outline the operation of the prototype for load balancing between access points.

\section{Scenario for Load Balancing in Multiaccess Environment}

Our experimentation scenario in wireless multiaccess environment is illustrated in Fig. 1. The scenario consists of two access networks (potentially with different technologies, e.g. WLAN and LTE), a service domain and a core network between them. Both access networks have one access point (AP), and these access networks have three different user devices connected to; User 1 (Video Streaming) and User 2 (Interfering Traffic) connected to AP1 and User 3 (Multiaccess Enhanced BitTorrent) connected to APs in both access network (AP1 and AP2).

We use a multiaccess BitTorrent [4] as a demonstration application for showing the advantages of the designed distributed resource management mechanisms. The multiaccess BitTorrent introduces the concept of a global - local tracker hierarchy for torrent-based content distribution systems to support localization of content distribution traffic and to enable the possibility for wireless end-users to use more than one network accesses at a time. The LocalTracker resides in the access network, as seen in the Fig. 1, and traces the peers in its own domain. The GlobalTracker is placed in the service domain and keeps a list of LocalTrackers being in this content distribution session. When an incoming peer joins to the content distribution session, it discovers its closest peers in the access network(s) by tracker hierarchy, and steers the download towards them. The system enables multiple connections to the network by a wireless user all the time, and this aspect is under consideration in this paper.

Distributed Decision Engine (DDE), similar to [5], is our approach for autonomous load balancing/resource management, and for the complexity problem introduced 
before. It is built on top of the idea of having a general distributed publish-subscribe message delivery system, which defines a common interface for different information producers, decision algorithms and configuration enforcement entities. The DDE enables autonomous cross-layer information exchange between different entities at different protocol layers and in different networks. The system implements an information caching functionality, in order to keep the latest information available and upto-date, as well as information processing for minimizing the data to be sent over the network. Furthermore, the DDE performs information filtering based on policies.

The DDE specification for a multiaccess capable access selection concentrates on 4 autonomous aspects: monitoring, network state analysis, decision making and configuration enforcement. In this scenario (cf. Fig. 1) the monitoring is implemented as DDE producers, which collect data from APs, user devices and localized services (LocalTracker). The network state is analysed by providing collected information to a fuzzy logic [6] based classification system that acts as a user for this information (DDE consumer). The result of the classification is provided back to the DDE (via producer interface) and delivered to the multiaccess BitTorrent client. The BitTorrent client receives the application specific result of the classification for each of the APs it is connected to. With this information the multiaccess client's built-in access selection mechanism decides whether to use a particular connection or not. Finally, the client does configuration enforcement and steers the download accordingly.

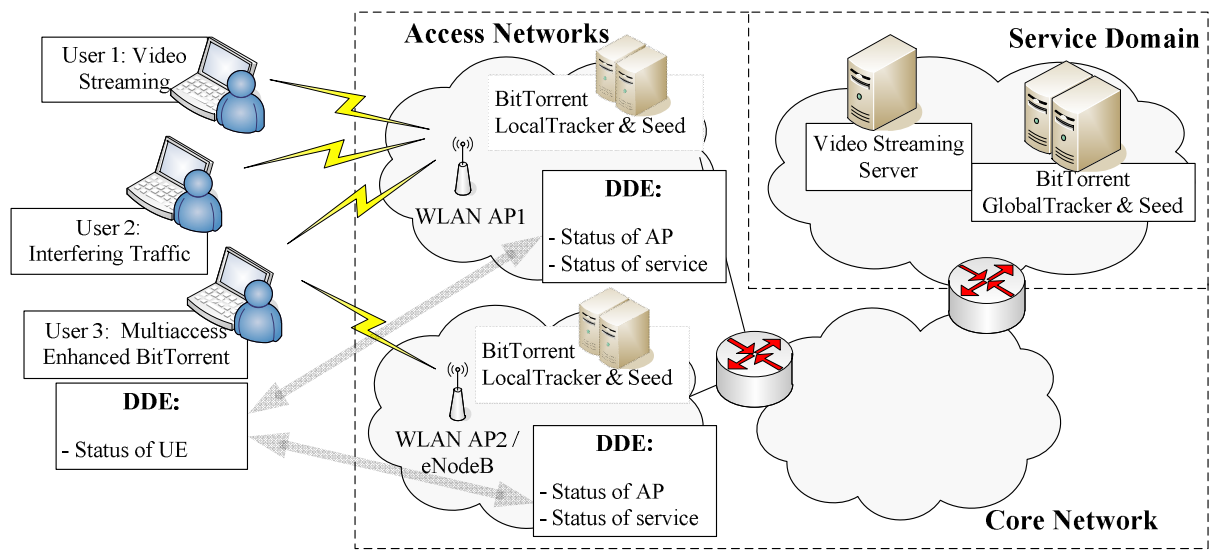

Fig. 1. Experimentation scenario for load balancing in multiaccess environment

The implemented proof-of-concept prototype with all the defined and implemented functionalities operates as follows. The User 1 is receiving a video stream over the AP1. The multiaccess and DDE enabled User 3 is retrieving content both over AP1 and AP2. Both users are satisfied with the QoS at this stage, since the video stream is solid and the download is proceeding rapidly. A third client, namely the User 2, connects also to AP1, and starts heavy traffic in order to emulate a huge increase in data traffic which in reality might be caused by several users using the same AP. In emerging networks, without autonomics, a service, i.e. the video stream would suffer from 
significantly decreased quality. With the help of DDE, the User 3 is aware of the congestion state in AP1, and balances the load between AP1 and AP2 by shifting the download only towards the AP2. Subsequently the traffic load in AP1 is decreased and the video stream quality returns to its original level. Finally, if the User 2 turns down interfering traffic, the User 3 moves back for using both APs. The preliminary tests are very promising and have already proved feasibility of getting significantly increased QoS in congestion situations. More evaluation is a part of the future work.

\section{Conclusions and Future Work}

In this paper we have presented our on-going work on load balancing. After describing a problem space and one of the validation scenarios of UMF, we gave a quick introduction into multiaccess enabled BitTorrent system, which is used as a demonstration application. Implemented prototype uses the Distributed Decision Engine for network node monitoring and autonomous decision making management, and it is applicable for multiaccess enabled access selection. Finally we outlined operation and the structure of implemented proof-of-concept prototype in practice.

As a part of the on-going and future work, our plan is to define and implement corresponding decision making process for service migration. The idea is to dynamically initiate service migration (e.g. LocalTracker \& Seed) closer to the user, based on the level of demand (from service domain towards the user). In this way operators, for instance, can formalize this local "caching functionality" during the big events in the certain area and would be able to reduce traffic load on their gateways.

Acknowledgments. This work has been supported by the UniverSelf project [2], partially funded by the European Community's Seventh Framework Programme and by the Intelligent Telecommunication Systems with Enhanced Cognitive Processing (ITSE) project, funded by VTT - Technical Research Centre of Finland.

\section{References}

1. Cisco Visual Networking Index: Global Mobile Data Traffic Forecast Update (2010-2015), http: / /www.cisco.com/go/vni

2. Univerself Project, http: / /www . univerself-project.eu/

3. Nguengang, G., et al.: UMF Specifications, Release 1. Univerself project deliverable D2.1 (2011),

http: / / www . univerself-project.eu/technical-reports

4. Pentikousis, K., Rautio, T.: A multiaccess network of information. In: IEEE International Symposium on World of Wireless Mobile and Multimedia Networks (WoWMoM), pp. 1-9 (June 2010)

5. Mäkelä, J., et al.: Distributed information service architecture for overlapping multiaccess networks. International Journal of Multimedia Tools and Applications (2011)

6. Zadeh, L.A., et al.: Fuzzy Sets, Fuzzy Logic, Fuzzy Systems. World Scientific Press (1996) ISBN 9810224214 